\title{
INFLUÊNCIA DA EMBALAGEM NA VIDA ÚTIL DE PRESUNTOS FATIADOS
}

\author{
Packing influence on the shelf life of sliced ham
}

\author{
Maria Cristina Bressan', Fabiane Lodi ${ }^{2}$, Milena Wolff Ferreira ${ }^{3}$, \\ Patrícia Lopes Andrade ${ }^{4}$, Cleube Andrade Boari ${ }^{5}$, Roberta H. Piccoli ${ }^{1}$
}

\begin{abstract}
RESUMO
Visou-se com este trabalho, comparar a vida útil de presunto fatiado sem capa de gordura (condicionadas à temperatura entre -1 e $4^{\circ} \mathrm{C}$ ), usando os tratamentos: sistema de embalagem a vácuo (EV) e sistema de embalagem com atmosfera modificada (AM) (50\% de $\mathrm{N}_{2}$ e $50 \%$ de $\mathrm{O}_{2}$ ), com os laminados flexíveis PET/PEBDL (AM1) e PVDC/EVA/PEBDL (AM2) sobre os parâmetros microbiológicos e físico-químicos, por 35 dias. As análises realizadas foram: contagem padrão em placas (CCP), contagem de fungos (CF), pH e umidade. Nas análises do dia inicial de trabalho verificou-se a contagem de $<10^{2} \mathrm{UFC} / \mathrm{g}$ de Staphylococcus aureus e para os demais microrganismos $<10^{1} \mathrm{UFC} / \mathrm{g}$. Com relação aos tratamentos, verificou-se que a embalagem com AM estendeu em 5 dias a vida útil do produto em relação a embalagem EV. O pH inicial médio de 6,4 aumentou para 6,55; 6,58 e 6,63 nos tratamentos EV, AM1 e AM2, respectivamente. $\mathrm{O}$ tratamento AM2 aumentou a vida útil em relação ao AM1.
\end{abstract}

Termos para indexação: Atmosfera modificada, polietileno, poliamida.

\begin{abstract}
The aim of this work was to compare the shelf-life of sliced ham without fat layer storage in a range of temperature between $1^{\circ}$ and $4^{\circ} \mathrm{C}$ using the following treatments: vacuum packing (VP) and packing with modified atmosphere (MA) $\left(50 \%\right.$ of $\mathrm{N}_{2}$ and $50 \%$ of $\mathrm{O}_{2}$ ) using flexible sheets of PEAD/LDPE (MA1) and PVDC/EVA/LDPE (MA2) on microbiological and physical chemical parameters for 35 days. Analysis of plate count of microorganisms, $\mathrm{pH}$ and humidity were conduced to evaluate the shelf-life of ham. In the first day of analyze the countings of Staphylococcus aureus had been inferior to $10^{2} \mathrm{CFU} / \mathrm{g}$ and for the other microorganisms inferior to $10^{1} \mathrm{CFU} / \mathrm{g}$. The packed with modified atmosphere increased five days in the shelf-life of the product when compared to vacuum packing. The initial $\mathrm{pH}(6.4)$ increased to $6.55,6.58$ and 6.63 on VP MA1 and MA2, respectively. The treatment MA2 increased the shelf-life when compared to modified atmosphere (1).
\end{abstract}

Index terms: Modified atmosphere, polyethylene, polyamide.

(Recebido em 8 de outubro de 2004 e aprovado em 16 de maio de 2006)

\section{INTRODUÇÃO}

Atualmente verifica-se crescente demanda dos consumidores por alimentos de melhor qualidade, de preferência frescos, naturais e práticos para seu preparo. Este interesse tem contribuído para o desenvolvimento de novos métodos para prolongar a vida útil dos produtos, associado à redução nos aditivos e conservantes artificiais, vistos com desconfiança pelos consumidores.

Alguns produtos cárneos apresentam maior probabilidade de deterioração, pois não possuem barreiras, tais como cortes frios fatiados e acondicionados a vácuo, presunto cozido e produtos embutidos, contra o crescimento de bactérias deteriorantes apesar da pasteurização e armazenamento à baixa temperatura, esses precisam ser rapidamente consumidos (KRÖCHEL, 1999).

Os produtos fatiados, de modo geral, são altamente perecíveis, pois apresentam teores de sal entre 2 a $4 \%, \mathrm{pH}$ maior que 6,0 e nitrito residual abaixo de 100 ppm (HOLLEY, 1997). Além disso, este tipo de produto é bastante manipulado e apresenta uma maior superfície de contato com o oxigênio, fator esse que influencia a vida útil dos alimentos em decorrência ao seu efeito químico, como a oxidação lipídica, e por permitir o crescimento de microorganismos aeróbios. Como solução para esses problemas, encontram-se os produtos industrializados fatiados acondicionados a vácuo ou sob atmosfera modificada.

A embalagem a vácuo, empregada no acondicionamento de peças inteiras ou pequenas porções, tem como objetivo proteger o produto cárneo do contato com o oxigênio do ar. O oxigênio favorece o crescimento de microrganismos aeróbios de alto potencial de deterioração, que alteram o odor, a cor e a aparência dos produtos cárneos, acarreta na rancidez oxidativa das gorduras, causa alterações nos pigmentos da carne e

\footnotetext{
'Professora do Departamento de Ciência dos Alimentos da Universidade Federal de Lavras/UFLA - Cx. P. 3037 - $37200-000$ - Lavras, MG bressan@ufla.br

${ }^{2}$ Aluna de Pós Graduação Lato sensu - Universidade Federal de Lavras/UFLA - Cx. P. 3037 - 37200-000 - Lavras, MG

${ }^{3}$ Aluna de Mestrado em Ciência dos Alimentos da Universidade Federal de Lavras/UFLA - Cx. P. 3037 - 37200-000 - Lavras, MG

${ }^{4}$ Aluna de Graduação em Medicina Veterinária/UFLA - Cx. P. 3037 - 37200-000 - Lavras, MG.

${ }^{5}$ Aluno de Doutorado em Ciência dos Alimentos da Universidade Federal de Lavras/UFLA - Cx. P. 3037 - 37200-000 - Lavras, MG
} 
destrói algumas vitaminas e aromas. Na ausência de oxigênio, as bactérias láticas predominam e causam menor alteração na qualidade das carnes, mesmo em altas contagens (SARANTÓPOULOS \& SOLER, 1991).

Os sistemas de acondicionamento em atmosfera modificada são concebidos para efetuar a troca da atmosfera original ao redor do produto por uma mistura de gases, de modo a prever e exercer controle sobre as alterações que ocorrerão no produto, na embalagem e na própria atmosfera gasosa, em decorrência da interação dos gases com o produto; respiração do produto e à permeabilidade do sistema de embalagem. O objetivo principal da modificação da atmosfera é preservar o frescor do produto do primeiro dia de processamento e estender o prazo de manutenção desse frescor e os atributos de qualidade durante sua vida útil maior (GOMES, 1998). De acordo com Mano et al. (2002), os gases mais comumente utilizados são: o gás carbônico, devido a sua atividade antimicrobiana, é o que compõe a maior parte da mistura de gases (DEVLIEGHERE et al., 1998), e o nitrogênio, que é usado apenas para completar o espaço disponível (SORHEIM et al., 1999).

$\mathrm{O}$ acondicionamento de produtos frescos (carne e peixe) em atmosfera modificada prolonga a vida útil desses alimentos (LÓPEZ-GÁLVEZ et al., 1995; MANO et al., 1999; PENNEY et al., 1993), porém, trabalhos que avaliam produtos fatiados são escassos.

O presunto fatiado, quando acondicionado em embalagem de alta permeabilidade ao oxigênio tem vida útil reduzida em 4 dias, e quando acondicionado à vácuo, pode aumentar em 25 dias e em embalagens com atmosfera modificada (AM) em 30 dias (BRODY, 1996). Entretanto, os diferentes laminados flexíveis utilizados em AM podem alterar o tempo de conservação.

O material utilizado para embalagem também pode variar permitindo maior ou menor permeabilidade do oxigênio. Os mais utilizados são Poliamida e Polietileno, e a combinação deles permite maior ou menor transmissão de oxigênio (YEN et al., 1988).

O objetivo desse trabalho foi verificar a influência de diferentes embalagens a vácuo e embalagem com atmosfera modificada de presunto fatiado estocado a baixas temperaturas sobre o desenvolvimento microbiológico e condições físico-químicas.

\section{MATERIAL E MÉTODOS}

O presunto (fatiado sem capa de gordura) foi fabricado de acordo com o memorial descritivo de empresa comercial do Estado do Paraná e embutido em embalagem de PA/PEBD (Polietileno de Baixa Densidade / Poliamida). As peças foram enformadas em formas especiais (dimensões: 1000 x 150 x $110 \mathrm{~mm}$ ), cozidas e resfriadas de acordo com os procedimentos previstos para esse produto.

Todas as etapas do processo (preparo da massa, embutimento, enformagem, cozimento, resfriamento, fatiamento, higienização das embalagens, acondicionamento e estocagem) foram realizadas de acordo com as normas previstas no manual das Boas Práticas de Fabricação e Armazenamento (BRASIL, 1997).

As peças de presunto, depois de resfriadas, foram fatiadas com o auxílio da fatiadeira automática (Dixie Union Verpackungssysteme 87437 Kempten/All, Romerstre 12), com espessura de $1,5 \mathrm{~mm}$. O fatiamento e o acondicionamento dos presuntos fatiados foram realizados em sala climatizada à temperatura constante de $4^{\circ} \mathrm{C}$.

As fatias de presunto, sobrepostas manualmente em cada embalagem (com capacidade de $100 \mathrm{~g}$, totalizando 180 embalagens para cada tratamento) foram acondicionadas em três tipos de embalagem:

-Tratamento controle: os produtos acondicionados à vácuo foram acondicionadas em sacos de material de baixa permeabilidade aos gases Poliamida e Polietileno de Baixa Densidade (PA/PEBD) e com dimensões de 180 x 210 x $0,20(\mathrm{~mm})$.

-Tratamento com Atmosfera Modificada 1 ( $\left.\mathrm{AM}_{1}\right)$ : o material de embalagem foi Polietileno Tereftalato e Polietileno de Baixa Densidade Linear (PET/PEBDL), com taxa de permeabilidade aos gases e vapor de água superior em relação a outra embalagem, com dimensões de 227 x 260 x $0,25 \mathrm{~mm}$.

-Tratamento com Atmosfera Modificada $2\left(\mathrm{AM}_{2}\right)$ : o material de embalagem foi Policloreto de Vinilideno/ Copolímero Etileno e Acetato de Vinila/ Polietileno de Baixa Densidade Linear (PVdC/EVA/PEBDL), e com dimensões de 130 x 200 x $0,15 \mathrm{~mm}$.

Com relação às embalagens, os valores para: Taxa de Permeabilidade ao Oxigênio para as diferentes estruturas foram: Polietileno/Poliamida/Polietileno $=65,00$ $\mathrm{cc} / \mathrm{m} 2 / \mathrm{dia} ;$ Poliéster $/$ Polietileno $=70,00 \mathrm{cc} / \mathrm{m} 2 / \mathrm{dia} ;$ Polietileno/EVA/Polietileno $=<3000 \mathrm{cc} / \mathrm{m} 2 / \mathrm{dia} ; \mathrm{e} \mathrm{EVA} /$ PVDC/EVA - 100,00 (cc/m2/dia); e para Taxa de Permeabilidade ao Gás Carbônico foram Polietileno/ Poliamida $/$ Polietileno $=186,00(\mathrm{cc} / \mathrm{m} 2 / \mathrm{dia}) ;$ Poliéster $/$ Polietileno $=350,00(\mathrm{cc} / \mathrm{m} 2 / \mathrm{dia}) ;$ Polietileno/Ava $/$ Polietileno $=<8000(\mathrm{cc} / \mathrm{m} 2 / \mathrm{dia}) ;$ EVA/PVDC/EVA $=30,00$ (cc/m2/dia). Esses dados ou valores foram obtidos com $0 \%$ umidade. $\mathrm{E}$ as gramaturas das diferentes estruturas foram: PEBD/PA 180 x 210 x 0,20=6,8 g. aprox. 7 g.; PET/ 
PEBDL 227 X 260 X 0,25 = 13,6 g. aprox. 14 g.; e PVDC/ EVA/PEBDL $130 \times 200 \times 0,15=3,6$ g. aprox. 4 g.

Nos tratamentos $\mathrm{AM}_{1}$ e $\mathrm{AM}_{2}$ foi usada uma composição de gases $\left(\mathrm{N}_{2} / \mathrm{O}_{2}\right)$ semelhante.

A estocagem dos produtos acondicionados foi efetuada em câmara de refrigeração mantida à temperatura entre $-1^{\circ} \mathrm{C}$ e $4^{\circ} \mathrm{C}$.

Para a realização das análises microbiológicas, foram retiradas diariamente das embalagens amostras de $25 \mathrm{~g}$ de presunto de cada tratamento e, em seguida, foi feita a homogeneização em $225 \mathrm{~mL}$ de água peptonada $0,1 \%$ (p/v) esterilizada em liquidificador doméstico durante um minuto.

As análises microbiológicas foram realizadas de acordo com a metodologia descrita pelo Método Oficial do Ministério da Agricultura (LANARA, 1981). Na análise de Contagem Padrão em Placas, utilizou-se o Plate Count Agar (PCA), incubou-se a $35^{\circ} \mathrm{C}$ por 48 horas. A caracterização inicial da amostra foi feita pela análise de Staphylococcus aureus, utilizando o Ágar Baird-Paker. Para a Contagem de Fungos, utilizou-se o Ágar Dextrose Batata com incubação a $25^{\circ} \mathrm{C}$ por cinco dias. Para a caracterização inicial da amostra foram feitas análises de Coliformes totais e termotolerantes, realizadas após incubação a $35^{\circ} \mathrm{C}$ por 24 horas, de acordo com a metodologia descrita pela AOAC (1990).

$\mathrm{O} \mathrm{pH}$ foi medido potenciometricamente (INSTITUTO ADOLFO LUTZ, 1985), na qual uma amostra de 10 gramas de presunto fatiado foi coletada aleatoriamente na embalagem e homogeneizada.

$\mathrm{O} \mathrm{pH}$ do produto foi determinado durante o período de estocagem, junto com a análise microbiológica. A umidade foi determinada durante o período de estocagem, utilizando o método de umidade volátil em estufa a $105^{\circ} \mathrm{C}$ por 4 horas.

\section{RESULTADOS E DISCUSSÃO}

A contagem padrão em placas (CPP) no primeiro dia do experimento demonstrou que as amostras do $\mathrm{AM}_{1}$ apresentaram contagem de $2 \times 10^{2} \mathrm{UFC} / \mathrm{g}$ valor superior aos produtos acondicionados a vácuo, $<10^{1} \mathrm{UFC} / \mathrm{g}$, e em $\mathrm{AM}_{2}, 2 \times 10^{1} \mathrm{UFC} / \mathrm{g}$. Isso indicou que as práticas de higienização e desinfecção e,ou, o controle de temperatura foram inadequados em alguma das fases do processamento, fatiamento ou embalagem do produto, de forma que o número de microrganismos mesófilos na fase inicial do armazenamento nos diferentes tratamentos não foi semelhante.

Os dados para CPP (FIGURA 1) mostram a evolução de crescimento de microrganismos aeróbios mesófilos durante os 35 dias de monitoramento, e quando submetidos à análise de regressão mostraram ajustamento eficiente a retas lineares. Os resultados para CPP apresentaram diferença $(\mathrm{P}<0,05)$, indicando que a evolução de crescimento de mesófilos em cada tratamento foi distinta. Como a legislação (Portaria $n^{\circ}$ 451 de 19/09/97 do MA) não apresenta limites microbiológicos para esse produto, foi considerado como CPP limite o valor de $10^{3} \mathrm{UFC} / \mathrm{g}$, adotados pela empresa. Assim, a vida útil dos produtos foi de 18, $21 \mathrm{e}$ 24 dias para presuntos fatiados submetidos a condições de embalagens de EV, $\mathrm{AM}_{1}$ e $\mathrm{AM}_{2}$, respectivamente. Esses resultados mostraram que os presuntos fatiados submetidos à AM apresentaram uma maior vida útil do que o produto EV, quando considerado os microrganismos aeróbios mesófilos. Quando considerado os tratamentos com atmosfera modificada, o $\mathrm{AM}_{2}$ (PVdC/EVA/PEBDL) apresentou condições de conservação mais eficientes, do que o AM (PET/ $_{1}$ PEBDL), embora a composição de gases de ambos tenha sido a mesma. Considerando os limites microbiológicos usados pela empresa, verificou-se que a embalagem a vácuo foi efetiva para o consumo até o $18^{\circ}$ dia de estocagem, alcançando 3,34 $\mathrm{log} \mathrm{UFC} / \mathrm{g} \mathrm{de}$ microrganismos aeróbios mesófilos. Ou seja, o produto fatiado acondicionado a vácuo pode ter sua qualidade assegurada, em termos microbiológicos, por 18 dias

García-Esteban et al. (2004), comparando duas embalagens com atmosfera modificada e embalagem a vácuo durante oito semanas, verificaram que, em ambas as embalagens, a contagem de aeróbios mesófilos foi inferior a $10^{4} \mathrm{UFC} / \mathrm{g}$, e que o número de colônias de microrganismos aeróbios mesófilos até a $8^{\mathrm{a}}$ semana aumentaram lentamente em relação ao número inicial observado, apresentando contagens de $7,5 \times 10^{2}$ e $9,45 \times 10^{3} \mathrm{UFC} / \mathrm{g}$ nas embalagens a vácuo; $7,4 \times 10^{3} \mathrm{e} 1,95 \times 10^{3} \mathrm{UFC} / \mathrm{g}$ nas embalagens com 100 $\%$ de nitrogênio; e, $1,65 \times 10^{4} \mathrm{e} 4,35 \times 10^{3}$ nas embalagens com $20 \%$ de gás carbônico e $80 \%$ de nitrogênio, no primeiro dia do experimento e após a oitava semana de estocagem, respectivamente.

$\mathrm{Na}$ contagem de fungos $(\mathrm{CF})$, o presunto, sob as condições do tratamento $\mathrm{AM}_{1}$ apresentou número de colônias de 2,9 $\times 10^{2} \mathrm{UFC} / \mathrm{g}$ superior aos tratamentos $\mathrm{EV}$ e $\mathrm{AM}_{2}$, cuja contagem foi de $<10^{1} \mathrm{UFC} / \mathrm{g}$ ). Utilizando embalagem a vácuo, $100 \% \mathrm{~N}_{2}$ e $20 \% \mathrm{CO}_{2}+80 \% \mathrm{~N}_{2}$, Fernando et al. (1995) encontraram valores menores para a contagem de fungos para o tratamento a vácuo $\left(<1,0 \times 10^{1}\right.$ $\mathrm{UFC} / \mathrm{g}$ ) e $<1,0 \times 10^{2} \mathrm{UFC} / \mathrm{g}$ para os dois tratamentos com atmosfera modificada. 
Comportamento semelhante foi observado para a contagem de Staphylococcus aureus, indicando que possivelmente tenha ocorrido maior contaminação no momento de embalagem das amostras do tratamento $\mathrm{AM}_{1}$.

As análises Coliformes totais e termotolerantes apresentaram contagem $<10^{1} \mathrm{NMP} / \mathrm{g}$ em todos os tratamentos, não sendo observado desenvolvimento dos mesmos ao longo dos 35 dias de estocagem.

A presença de fungos em presunto fatiado é indesejável, pois seu desenvolvimento acarreta alterações no odor, sabor e pode promover o estufamento da embalagem (SARANTÓPOULOS \& SOLER, 1991). Como a legislação (Portaria n ${ }^{\circ} 451$ de 19/09/1997 do MAPA) não apresenta limites microbiológicos para esse produto, adotou-se o limite inferior a $10^{2} \mathrm{UFC} / \mathrm{g}$. $\mathrm{O} \mathrm{AM}_{2}$ mostrou valores reais menores que $10^{2} \mathrm{UFC} / \mathrm{g}$ até o $24^{\circ} \mathrm{dia}$, enquanto que no tratamento $\mathrm{EV}$ e $\mathrm{AM}_{1}$ contagem inferior $10^{2} \mathrm{UFC} / \mathrm{g}$ foi mantida até o $14^{\circ}$ dia, ou seja houve diferença de 10 dias de vida útil (FIGURA 2).

A redução no tempo de vida útil encontrada pode ser atribuída, nos tratamentos $\mathrm{EV}$ e $\mathrm{AM}_{1}$, a parcial permeabilidade aos gases e a perda de maior quantidade de $\mathrm{CO}_{2}$ ao meio, ocasionando o crescimento de fungos.
Por outro lado, no tratamento com $\mathrm{AM}_{2}$ houve menor perda de $\mathrm{CO}_{2}$ e, consequientemente maior controle no desenvolvimento de fungos. Analisando as médias obtidas dos 35 dias detectou-se diferença $(\mathrm{P}<0,05)$ a partir do $28^{\circ}$ dia.

Ao se iniciar o experimento, o produto apresentou $\mathrm{pH}$ de 5,4 com aumento durante o tempo de armazenamento (FIGURA 3). Possivelmente tal comportamento é resultado do crescimento de microrganismos proteolíticos. Entre os resultados de $\mathrm{pH}$ do diferentes tratamentos ao longo do tempo não houve diferença.

Mano et al. (2002), estudando o efeito da embalagem sob atmosfera modificada e a vácuo em carne suína, não observaram variações no $\mathrm{pH}$, provavelmente estes resultados devam-se à capacidade tampão dos componentes da carne, que podem ser suficientes para inibir o efeito do $\mathrm{CO}_{2}$ solubilizado.

Como consequiência do $\mathrm{CO}_{2}$ solubilizado, presente nas embalagens com atmosfera modificada, Mcmuller \& Stiles (1991) encontraram diminuição do pH durante o tempo de estocagem de carne suína. No presente trabalho tal solubilidade pode não ter apresentado influência devido ao processo de cozimento do presunto e armazenamento sob refrigeração.

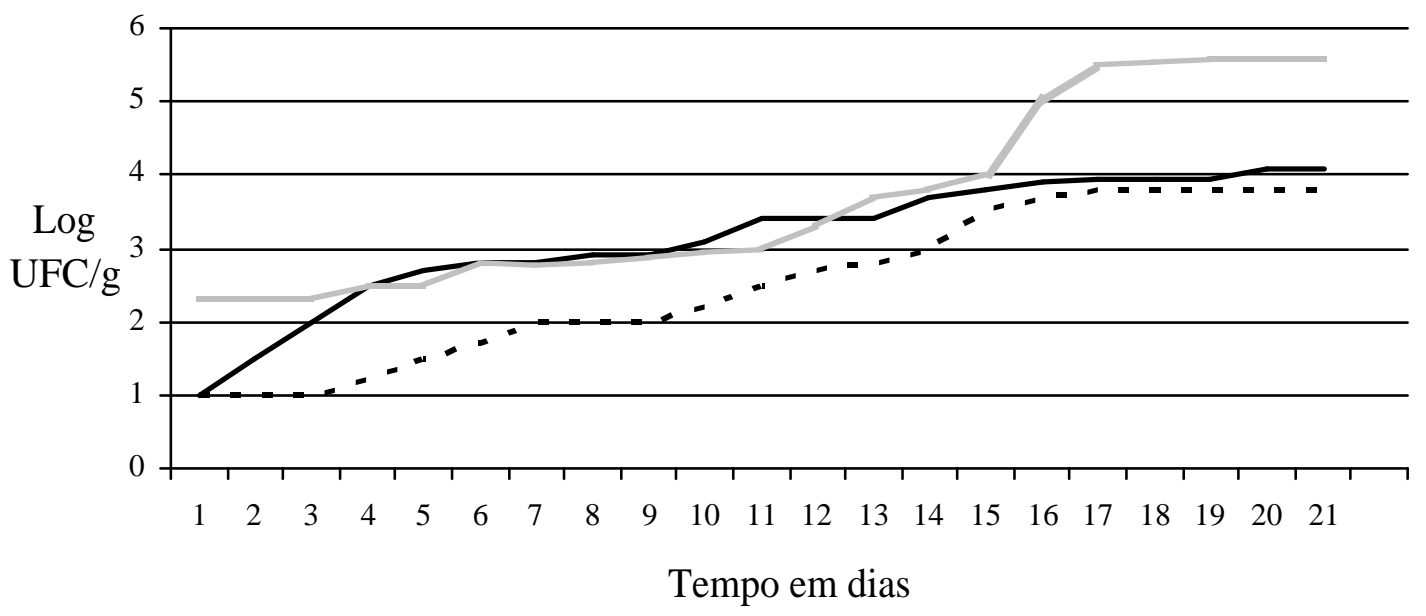

—Vácuo $\longrightarrow$ AM1 - - - AM2

FIGURA 1 - Evolução do crescimento de microrganismos aeróbios mesófilos nas diferentes embalagens utilizadas. 


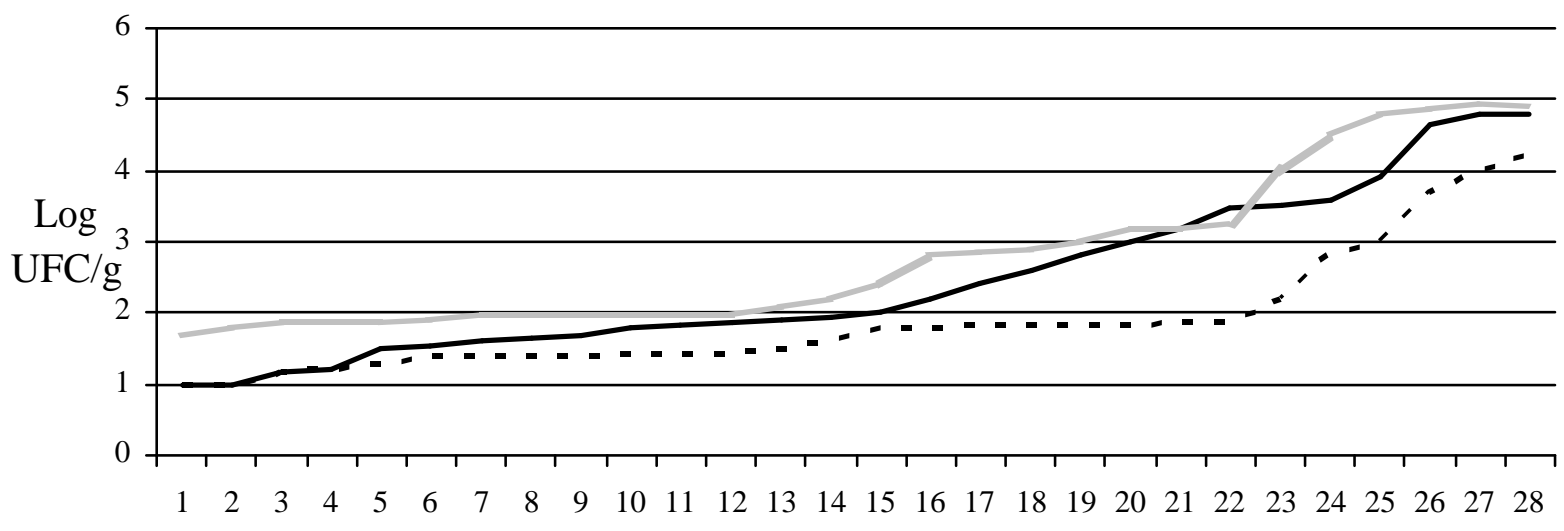

Tempo em dias

$$
\text { — Vácuo —AM1 - - - AM2 }
$$

FIGURA 2 - Evolução do crescimento de fungos durante 28 dias de armazenamento a $4^{\circ} \mathrm{C}$ submetidos a diferentes tratamentos.

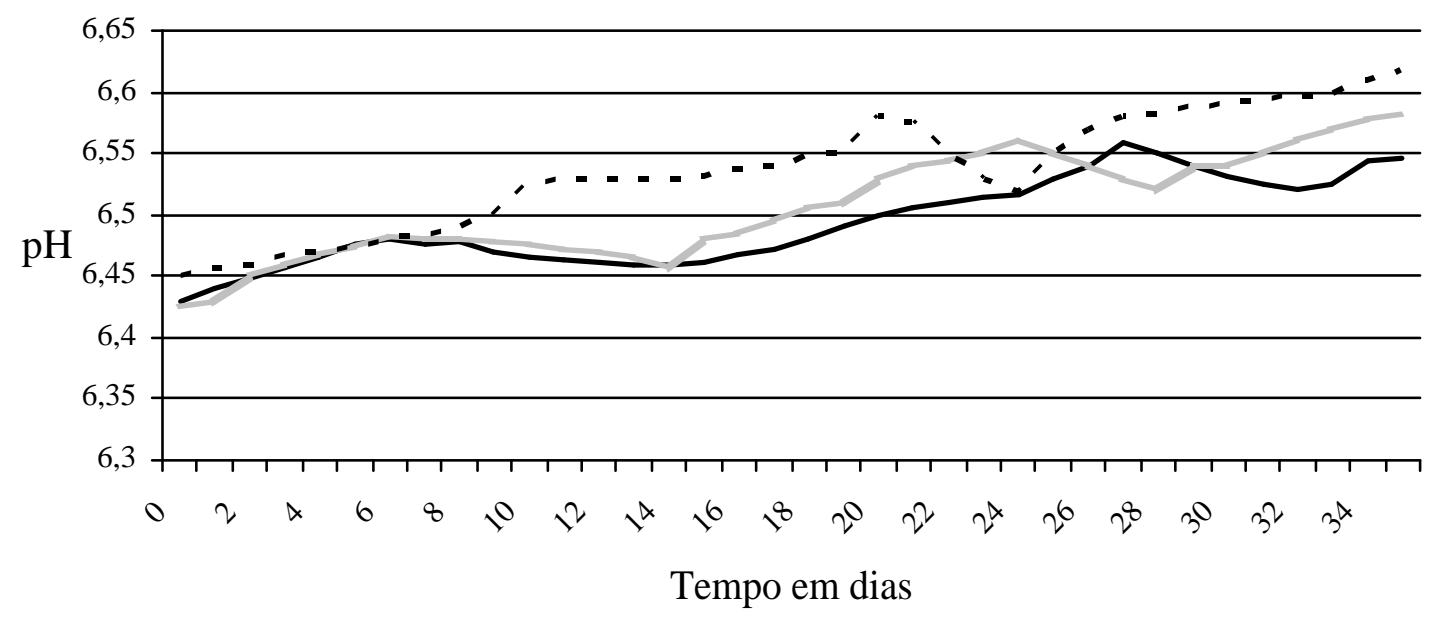

$$
\text { — Vácuo } \quad \text { AM1 - - - AM2 }
$$

FIGURA 3 - Comportamento do pH dos presuntos sob diferentes tratamentos durante 35 dias de armazenamento.

\section{CONCLUSÕES}

O sistema de embalagem em que utilizou-se atmosfera modificada estendeu por cinco dias a vida útil do presunto fatiado em relação ao sistema de embalagem a vácuo. E no sistema com atmosfera modificada, a vida útil do presunto acondicionado em
PVdC/EVA/PEBDL foi superior a vida útil do produto acondicionado em PET/PEBDL.

\section{AGRADECIMENTOS}

FAPEMIG.

Os autores agradecem ao CNPq, CAPES e

Ciênc. agrotec., Lavras, v. 31, n. 2, p. 433-438, mar./abr., 2007 


\section{REFERÊNCIAS BIBLIOGRÁFICAS}

ASSOCIATION OF OFFICIAL ANALYTICAL CHEMISTS, Official methods of analysis of the Association of Analytical Chemists. 15. ed. Arlington, 1990.

BRASIL. Portaria no 368, de 4 de setembro de 1997 do Ministério da Agricultura, Abastecimento e Pecuária. Dispõe sobre regulamento técnico sobre as condições higiênico-sanitárias e de boas práticas de fabricação para estabelecimentos elaboradores/industrializados de alimentos. Brasília, DF, 1997.

BRODY, A. L. Envasado de alimentos em atmosferas controladas, modificas y a vacio. Zaragoza: Acribia, 1996. $388 \mathrm{p}$.

DEVLIEGHERE, F.; DEBEVERE, J.; IMPE, J. van. Concentration of carbon dioxide in the water-phase as a parameter to model the effect of a modified atmosphere on microorganisms. International Journal of Food Microbiology, Amsterdam, v. 43, p. 105-113, 1998.

FERNANDO, G. D. G. de; NYCHAS, G. J. E.; PECK, M. W.; ORDÓÑOZ, J. A. Growth/survival of psychotrophic pathogens on meat packaged under modified atmosphere. International Journal of Food Microbiology, Amsterdam, v. 28, p. 211-231, 1995.

GOMES, C. T. Sistemas de conservação com atmosfera modificada. Revista Nacional da Carne, São Paulo, n. 259, p. 79-80, 1998.

GRACÍA-ESTEBAN, M.; ANSORENA, D.; ASTIASARÁN, I. Comparison of modified atmosphere packaging and vacuum packaging for long period storage of dry-cured ham: effects on color, texture and microbiological quality. Meat Science, Barking, v. 67, p. 57-63, 2004.

HOLLEY, R. A. Impact of slicing hygiene upon shelf life and distribution of bacteria in vaccum packaged cured ham. Food Microbiology, Winnipeg, v. 14, p. 201-211, 1997.

INSTITUTO ADOLFO LUTZ. Normas analíticas do Instituto Adolfo Lutz: métodos químicos e físicos para análises de alimentos. 3. ed. São Paulo, 1985. v. 1, 533 p.

KRÖCHEL, L. Natural barriers for use in biopreservation. Flesch Wirtschat International, Frankfrurt, n. 2, p. 36-38, 1999.
LANARA. Métodos analíticos oficial para controle de produtos de origem animal e seu ingredientes. Brasília, DF: [s.n.], 1981. Tomo 1-2.

LÓPEZ-GÁLVEZ, D.; HOZ, L.; ORDÓÑEZ, J. A. Effect of carbon dioxide and oxygen enriched atmospheres on microbiological and chemical changes in refrigerated tuna (Thunnus alalunga) steaks. Journal of Agriculture of Food Chemistry, Easton, v. 43, p. 483-490, 1995.

MANO, S. B.; ORDÓÑEZ, J. A.; FERNANDO, G. D. G. de. Aumento de la vida útil y microbiologia de la carne de pavo envasada en atmósferas modificadas. Revista Brasileira de Ciência Veterinária, [S.1.], v. 6, n. 2, p. 55-65, 1999.

MANO, S. B.; PEREDA, J. A. O.; FERNADO, G. D. G. Aumento da vida útil e microbiológica da carne suína embalada em atmosfera modificada. Ciência e Tecnologia de Alimentos, Campinas, v. 22, n. 1, p. 1-10, jan./abr. 2002.

McMULLER, L. M.; STILES, M. E. Changes in microbial parameters and gás composition during modified atmosphere storage of fresh pork loin chops. Journal of Food Protection, Des Moines, v. 54, p. 778-783, 1991.

PENNEY, N.; HAGYARD, C. J.; BELL, R. G. Extension of shelf-life of chlled Alice roast beef by carbon dioxide packaging. International Journal of Food Science Tecnology, [S.l.], v. 28, p. 181-191, 1993.

SARANTÓPOULOS, C.; SOLER, R. Embalagens com atmosfera modificada/controlada. Revista Nacional da Carne, São Paulo, n. 209, p. 32-42, 1991.

SARANTÓPOULOS, C.; SOLER, R. Sistemas de acondicionalmento em embalagem de atmosfera modificada. Revista Nacional da Carne, São Paulo, n. 213, p. 16-19, 1994.

SORHEIM, O.; NISSEN, H.; NESBAKKEN, T. The storage life of beef and pork packaged in an atmosphere with low carbon monoxide and high carbon dioxide. Meat Science, Barking, v. 52, p. 157-164, 1999.

YEN, J. R.; BROWN, R. B.; DICK, R. L.; ACTON, J. C. Oxygentransmission rate of packaging films and light exposure effects on the colour stability of vaccumpackaged dry salami. Journal of Food Science, Chicago, v. 53, p. 1043-1046, 1988. 